\title{
Small-Bowel Polyps in Peutz-Jeghers Syndrome: Diagnosis by Wireless Capsule Endoscopy
}

G. D. De Palma, M. Rega, P. Ciamarra,

E. Di Girolamo, F. Patrone,

L. Mastantuono, I. Simeoli

Dept. of Surgery and Advanced

Technologies, Diagnostic and Therapeutic Endoscopy Section, Federico II University School of Medicine, Naples, Italy

\section{Corresponding Author}

\section{G. D. De Palma, M.D.}

Via De Gasperi, 7 80033 Cicciano (Napoli)

Italy

Fax: + +39-81-8262866

E-mail: gdepalma@arrotino.it
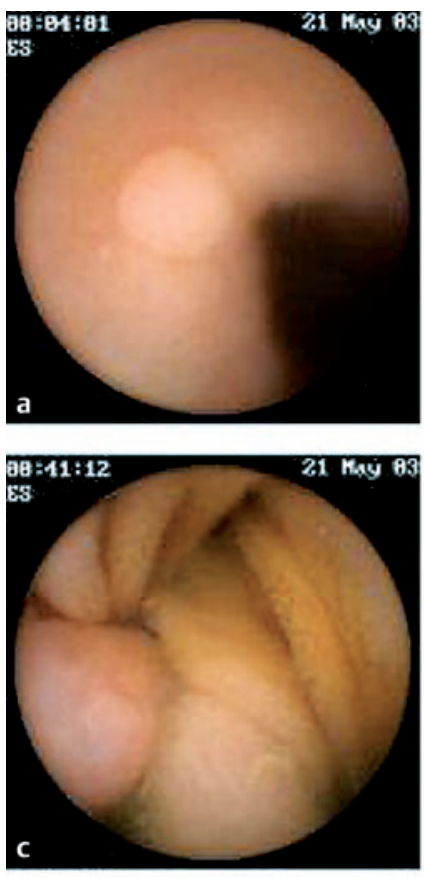

b
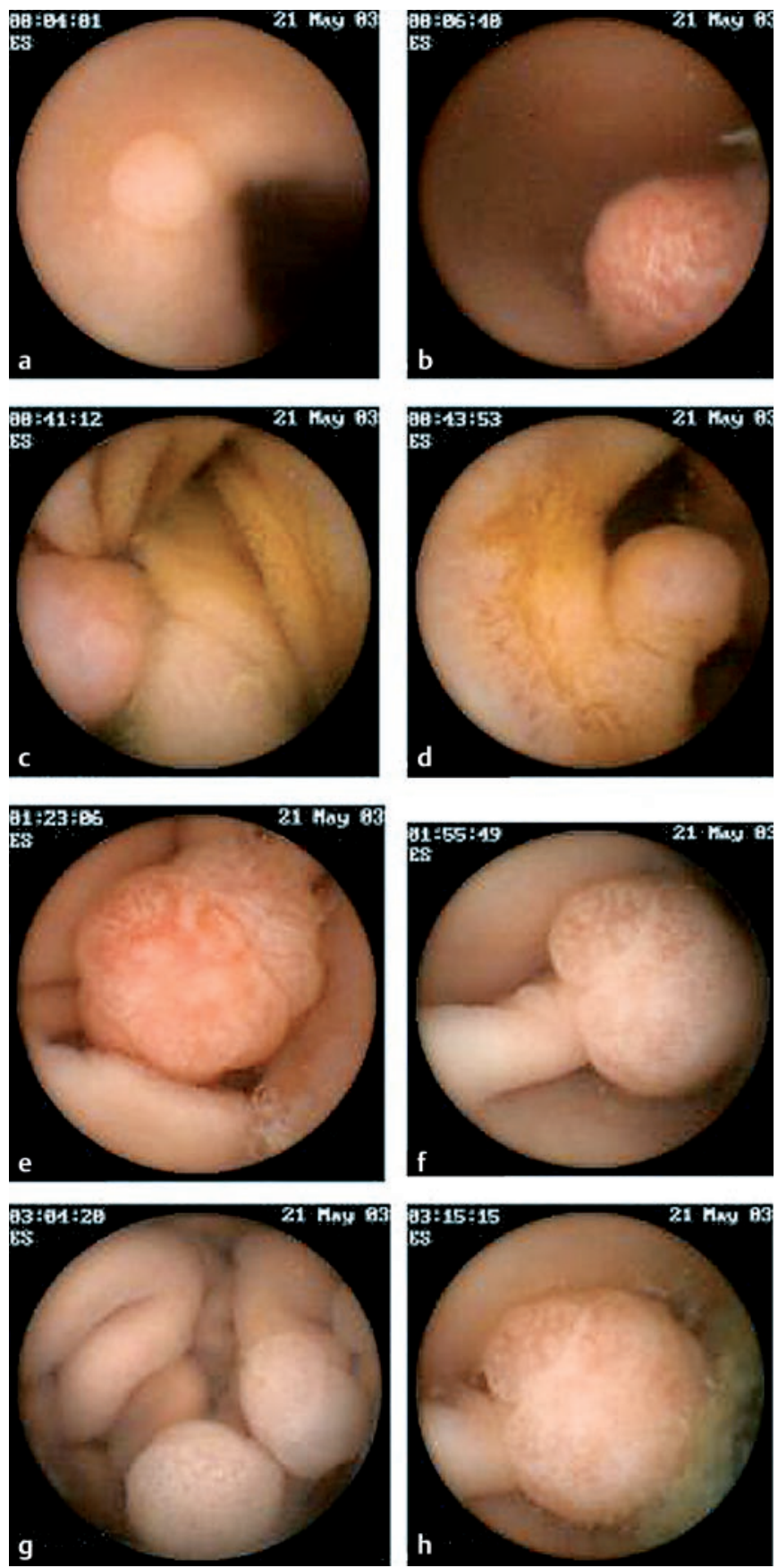

21. BNY $\mathrm{G}$
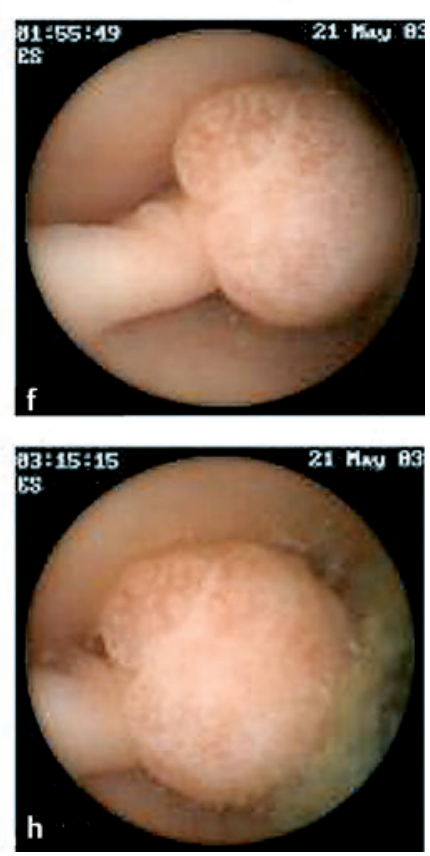

Figure 1 a, b Gastric polyps. c- $\mathbf{h}$ Jejunal polyps. A 14-year-old girl was referred for evaluation of abdominal pain and iron-deficiency anemia. The physical examination revealed evidence of mucocutaneous pigmentation. A capsule endoscopy examination was carried out, as she declined conventional imaging work-up. Capsule endoscopy revealed multiple polyps in the stomach and proximal jejunum. The polyps ranged from $1-2 \mathrm{~mm}$ to $2-3 \mathrm{~cm}$ in size, and the majority of them were pedunculated. Surgical enteroscopy was carried out with the patient under general anesthesia. All of the resected polyps had the histological features of hamartomas, with no adenomatous changes and no evidence of malignancy. 